\title{
Desempeño del Kit ELISA Chagas IICS V.1 para el diagnóstico de la enfermedad de Chagas
}

Laura Aria, María Eugenia Acosta, Yvalena Guillen, Alejandra Rojas, Teresa Meza, Belén Infanzón.

Departamento de Producción. Instituto de Investigación en Ciencias de la Salud. Universidad Nacional de Asunción, Paraguay

Cómo referenciar este artículo/

How to reference this article:
Aria L, Acosta ME, Guillen Y, Rojas A, Meza T, Infanzón B. Desempeño del Kit ELISA Chagas IICS V.1 para el diagnóstico de la enfermedad de Chagas. Mem. Inst. Investig. Cienc. Salud. 2016; 14(3): 7-13

\section{R E S U M E N}

En Paraguay, el tamizaje serológico para la enfermedad de Chagas en bancos de sangre es necesario, por lo cual es importante un método diagnóstico con alta sensibilidad. El ELISA Chagas test IICS V.1 es un ELISA indirecto sensibilizados con antígeno soluble de epimastigote de T.cruzi de la cepa Ypsilon. El objetivo del presente trabajo fue evaluar el desempeño del ELISA Chagas test IICS V.1 en comparación con kits comerciales, ademásanalizar los resultados de la evaluación externa e interna de calidad del kit. En este estudio observacional de prueba diagnóstica se analizaron 56 muestras de suerospositivos y negativos para antígenos de Trypanosoma cruzi, testados por el ELISA BiosChile, obteniéndose una concordancia excelente entre el ELISA Chagas test IICS V.1y los kits comerciales: Chagatest ELISA-Wiener, con Índice kappa: 0,89 IC de 95\% (0,76-1) y Test ELISA para Chagas III-Grupo BiosChile con Índice kappa: 0,92 IC 95\% (0,82-1).En la evaluación externa de calidad realizada por la Fundação Pró-Sangue/Hemocentro de São Paulo, Brasil en el periodo 2001 al 2012 se analizaron 450 muestras: 372 negativas y 78 positivas para $T$. cruzi, obteniéndose en dicha evaluación la calificación " $A$ " que indica ausencia de falsos positivos y negativos. Además, en el mismo periodo los valores del control interno se encontraron dentro del rango permitido de $\pm 2 \mathrm{DS}$. Los resultados obtenidos en el estudio demuestran la alta calidad de este test de producción nacional, que sumado al bajo costo del mismo, pueden ser utilizados en trabajos de campo, donde no necesita de instrumentación y las lecturas pueden realizarse a simple vista, constituyendo una herramienta válida y útil para el apoyo al diagnóstico de la enfermedad de Chagas.

Palabras claves: Chagas, T. cruzi, programa de evaluación, control interno.

\section{ELISA Chagas test IICS V.1 evaluation for the diagnosis of Chagas disease}

\section{A B S T R A C T}

In Paraguay, the serological screening for Chagas disease is mandatory in pregnant women and blood banks, therefore a high sensitivity diagnostic method is required. The aim of this study was to evaluate the ELISA Chagas test IICS V.1 by comparison with commercial kits and to analyze the external and internal quality evaluation results. In this descriptive observational study, 56 seropositive and seronegative to Trypanosoma cruzi samples were analyzed, obtaining an excellent concordance between the ELISA Chagas test IICS V.1 and these commercial kits: Chagatest ELISA-Wiener, Argentina (kappa index: 0.89) and Test ELISA Chagas III-GrupoBios, Chile (kappa index: 0.92).In the external quality assessment carried out by the Fundação Pró-Sangue /Blood Center of São Paulo, Brazil in the period 2001 to 2012, 450 samples were analyzed: 372 seronegative and 78 seropositive for $T$. cruzi. In this evaluation, an " $A$ " score was obtained indicating the absence of false positives and negatives. Additionally, in the same period of time the internal control values were within the accepted range of $\pm 2 S D$, with a confidence interval of $95 \%$. The results obtained in the present study demonstrate the high quality of this

Fecha de recepción: septiembre 2016. Fecha de aceptación: noviembre2016

Autor correspondiente: Laura Aria. Departamento de Producción. IICS

E-mail: lauraariazaya@yahoo.es 
locally produced test which added to its low cost, making it a valid and useful tool to support the diagnosis of Chagas disease.

Keywords: Chagas, T. cruzi, evaluation program, internal control.

\section{INTRODUCCIÓN}

La enfermedad de Chagas es una zoonosis causada por el hematozoario Trypanosoma cruzi. Es una endemia de muy amplia distribución afectando a 21 países de América especialmente a poblaciones rurales siendo un factor restrictivo para el pleno desarrollo económico de la región (1).

Se transmite al humano y otros mamíferos a través de insectos triatomíneos infectados, transfusión de sangre infectada, por vía transplacentaria, trasplante de órganos, vía digestiva entre otras. Los reservorios del parásito son mamíferos domésticos y silvestres (2$5)$. Se ha reportado ocasionalmente contagios por ingestión de sustancias contaminadas o infección accidental en el laboratorio (6).

La Organización Mundial de la salud (OMS)en los Estándares de Trabajo para Servicios de Sangre indica como norma que los servicios de sangre examinen como mínimo cada donación determinando anticuerpos contra $\operatorname{T}$.cruzi $(7,8)$.

Se recomienda que al menos, se realicen dos pruebas en paralelo que utilicen antígenos o principio distinto, debido a la diversidad inmunogénica de las diferentes cepas del parásito, la respuesta inmunitaria de los pacientes y la existencia de reacciones cruzadas con otros tripanosomatidos que coexisten en las áreas endémicas (9).

En Paraguay, siguiendo con esta normase realiza el tamizaje de anticuerpos contra T.cruzi en todos los donantes en bancos de sangre, mediante la determinación de la presencia de anticuerpos IgG contra $T$. cruzi por varios métodos, entre ellos el ELISA (10).

Debido a la alta prevalencia de la enfermedad de Chagas y el riesgo de adquirir una infección por $T$. cruzi a través de una transfusión de sangre de donantes infectados impulsó al desarrollo de un kit de ELISA para el diagnóstico de la enfermedad a nivel local en el Instituto de Investigaciones en Ciencias de la Salud de la Universidad Nacional de Asunción con el apoyo de la Cooperación Técnica Alemana (GTZ) (11). Este kit es el único de producción nacional y se viene utilizando en el país desde 1987 inclusive a nivel nacional por el Programa de Control de la Enfermedad de Chagas para el tamizaje en áreas endémicas (12) y también para el control de la transmisión congénita (13). El Chagas test IICS fue evaluado junto con otros kits comerciales en 1994 en el banco de sangre de Chile demostrando una excelente especificidad y sensibilidad mencionando que el ELISA es el test recomendado para la detección de Chagas. (14)

Para validar la calidad de los resultados obtenidos en el diagnostico serológico es esencial implementar controles tanto internos como externos, además el laboratorio debe realizar una elección adecuada de los test de diagnósticos antes de implementar su utilización en la rutina diagnóstica diaria $(15,16)$. El ELISA Chagas test IICS V.1 ha sido evaluado en el Programa de Control de Calidad Externo en Serología (PCECS)de la Fundação PróSangue/Hemocentro de São Paulo Brasil donde se mide el desempeño de los laboratorios que hacen el tamizaje serológico en bancos de sangre desde el año 1996, con 2 evaluaciones al año. La herramienta de trabajo es un panel de sueros que se envía a todos los participantes del programa para ser procesado como si fuese un conjunto de muestras. (17)

Cuando el tamizaje serológico lo ejecutan de modo adecuado profesionales capacitados, siguiendo las buenas prácticas de laboratorio y utilizando pruebas diagnósticas de buena calidad e instrumentos bien calibrados, se obtienen resultados precisos y confiables, capaces de mantener el riesgo de transmisión de enfermedades dentro de límites aceptables (17).

Entre las múltiples ventajas de la utilización del ELISA Chagas test IICS V.1 está su alta sensibilidad y especificidad $97 \%$ y $99 \%$ respectivamente evidenciado en un estudio realizado por la Fundação Pró-Sangue/Hemocentro de São Paulo, Brasil en donde se comparó varios kits provenientes de países de América Latina, con sueros caracterizados positivos y negativos por distintos métodos (18), más la garantía de un suministro continúo dado que es de producción local, debemos destacar también que pueden ser utilizados en trabajos de campo, donde no necesita de instrumentación y las lecturas pueden realizarse a simple vista.

Dado la diversidad de kits empleados para el diagnóstico de la enfermedad de Chagas en los laboratorios públicos y privados del país, se realizó el presente trabajo que tuvo como 
objetivo comparar el ELISA Chagas test IICS V.1 con kits comerciales y analizar los resultados de la evaluación externa e interna del kit. ELISA Chagas test IICS V.1

\section{MATERIALES Y METODOS}

Diseño del estudio: observacional de pruebas diagnósticas en el que se comparó el desempeño del Kit Chagas test-IICS V.1 con otros dos kits comerciales: BiosChile Test ELISA y Chagatest ELISA recombinante v 3.0 de Wiener.

\section{Comparación con kit comerciales}

Se seleccionaron 56 sueros de pacientes proveídos por el Centro Nacional de Trasfusión Sanguínea: 33 positivos y 23negativospara T. cruzi, por el ELISA BiosChile para Chagas. Los sueros debidamente codificados se encontraban almacenados en la seroteca del departamento de Producción.

Los kits comerciales utilizados fueron ELISAs indirectos BiosChile Test ELISA para Chagas III ${ }^{\circledR}$ (Laboratorios BiosChile, Santiago-Chile y Chagatest ELISA recombinante v 3.0 (Laboratorios Wiener, Rosario-Argentina).Todos los ensayos se realizaron siguiendo las instrucciones de los fabricantes de cada kit. La reactividad de cada una de las muestras se determinó según las especificaciones técnicas de cada kit.

El kit Chagas test-IICS V.1esunELISA indirecto que detecta IgG anti-T. cruzi, utiliza placas de 96 pocillos, que consisten en 12 tiras individuales con 8 pocillos(NUNC $\Re$ ) sensibilizadas con antígeno soluble de epimastigote de T.cruzi de la cepa Ypsilon. Las muestras y controles se emplean en una dilución 1/50 tampón de incubación (PBS)y se incuban por $30 \mathrm{~min}$. a temperatura ambiente (TA). Después se lavan dos veces con tampón de lavado (PBSTween) y se añade el conjugado anti IgG-POD y se vuelve a incubar $30 \mathrm{~min}$. aTA. Posteriormente se lavan tres veces con tampón de lavado y se agrega el sustrato ABTS (2,2- azino-bis (3 ethylbenzothiazoline 6 sulfonicac) diammoniumsalt) incubándose por 20 min. a TA y se detiene la reacción con Teepol 610. La lectura de la densidad óptica se realiza en el lector de ELISA a $405 \mathrm{~nm}$ (BioRad 550) pero es posible discriminar mediante lectura visual. (11).

La concordancia entre el kit Chagas test-IICS V.1 de producción local y los kits comerciales, y, entre los dos kits comerciales, se estableció mediante los valores del índice kappa calculado con el paquete estadístico Epi-dat versión 3.1 para Windows. La interpretación de los valores del índice kappa se realizó según las recomendaciones de Landis y Koch: concordancia pobre $=0$, leve entre 0 y 0,20 , baja entre 0,21 y 0,40 , moderada entre 0,41 y 0,60 , buena entre 0,61 y 0,80 , casi perfecta entre 0,81 y $1,00 .(19)$

\section{2-Evaluación externa}

Con el apoyo de la OPS se desarrolló el Programas de control externo de la calidad en serología en América Latina desde 1994 (17), donde el IICS participa desde 1996. Las 450 muestras utilizadas en este estudio provenían de multipaneles de sueros del "Programa de Evaluación Externa de Desempeño en Serología" de la Fundação Pró-Sangue/ Hemocentro de São Paulo de los años 2001 al 2012. Cada evaluación estaba compuesta por 24 muestras de sueros con diferentes reactividades a los marcadores obligatorios en el tamizaje serológico de donantes de sangre (Chagas, Sífilis, Hepatitis B y C, HIV, HTLV) incluyendo muestras negativas para estas patologías.

La producción de los multipaneles fue realizada en el Departamento de Control de la Calidad de la Superintendencia de Serología de la Fundação Pró-Sangue/Hemocentro de São Paulo, por recalcificación de plasma, utilizando Bronidox-L como conservante. Se utilizaron diversas pruebas de marcas diferentes, entre ellas pruebas de confirmación, de modo que el grado de seguridad para caracterizar cada muestra como positiva o negativa fuese lo mayor posible. (15)

El Programa de Evaluación Externa utilizó los siguientes criterios para la evaluación:

A: Resultados correctos, sin resultados Falsos Positivos y Falsos Negativos

B1: Fueron observados resultados "Falsos positivos" $(<5 \%$ del total de determinaciones efectuadas)

B2: Fueron observados resultados "Falsos positivos" ( $>5 \%$ del total de determinaciones efectuadas)

C: Detección de resultados Falsos Negativos 


\section{3-Evaluación interna}

El control interno evalúa la precisión del kits (20). Se utilizó un suero control interno de baja reactividad que consiste en un pool de sueros pertenecientes a donantes que fueron testados como positivos para $T$. cruzi en el tamizaje serológico de los donantes y descartados por el banco de sangre del Centro Nacional de Transfusión Sanguínea, teniendo en cuenta los criterios de aceptación:

El índice de relación de positividad que es la relación entre la densidad óptica (DO) y el punto de corte $(\mathrm{CO})$ debe presentar: $\mathrm{DO} / \mathrm{CO}>1$ entre un rango recomendado de 2,0 a 4,5. (21)

La carta control Levey-Jennings fue construida con los valores de DO de 20 corridas de diferentes días. Los valores de los resultados del suero control interno se incorporaron diariamente en la carta control. Los valores de DO obtenidos se promediaron por año y estos fueron marcados en la carta control para ser evaluados del 2008 al 2012.

Se analizaron los valores del promedio de DO obtenidos del suero control interno por las reglas de Shewhart que expresan que aproximadamente el 95\% de los resultados tienen que encontrarse dentro del intervalo \pm 2DS para decidir si se acepta la corrida (22).

Para la precisión interensayo del kit Chagas test-IICS V.1 se determinó el CV (coeficiente de variación) por año, con el criterio de aceptación de que el mismo no debe superar el $20 \%(23)$.

\section{RESULTADOS}

Se realizó la comparación del kits Chagas test IICS V.1 con los kits comerciales con 56 sueros positivos y negativos para la enfermedad de Chagas, obteniéndose una concordancia con el Chagastest ELISA (Wiener) de 0.89 con IC (intervalo de confianza) de $95 \%(0,76-1)$ y con el BiosChile Test ELISA para Chagas III de 0,92 con IC 95\% $(0,82-1)$, que indica una concordancia casi perfecta o excelente entre 0,81 y 1,00 según el Índice kappa de Landis y Koch, en cuanto a la concordancia entre BiosChile Test ELISA para Chagas III y el Chagastest ELISA (Wiener) es de 0.85 con IC 95\%(0.6-1.1). En laTabla 1 se observan las concordancias obtenidas entre los kits estudiados. En el figura 1 se muestra las desviaciones ópticas (DO) obtenidas del Chagastest ELISA (Wiener), BiosChile Test ELISA para Chagas III y el kit Chagas test-IICS, se observa una DO de los sueros positivos, relativamente mayor en el Chagastest ELISA (Wiener) con respecto a BiosChile Test ELISA para Chagas III y el kit Chagas test-IICS V.1, en cambio los DO de los sueros negativos tienen un comportamiento similar en los tres kits, aunque se observan algunos discordantes.

Tabla 1. Comparación entre el Chagas test-IICS y otros kits comerciales

\begin{tabular}{|c|c|c|c|c|}
\hline & & \multicolumn{2}{|c|}{ Chagas test-IICS } & \multirow{2}{*}{ Total } \\
\hline & & Positivo & Negativo & \\
\hline \multirow{2}{*}{ WIENER } & Positivo & 32 & 2 & 34 \\
\hline & Negativo & 1 & 21 & 22 \\
\hline \multirow{2}{*}{ BIOS CHILE } & Positivo & 32 & 1 & 33 \\
\hline & Negativo & 1 & 22 & 23 \\
\hline Total & & 33 & 23 & 56 \\
\hline
\end{tabular}

Concordancia: BiosChile-IICS: 0,92 IC 95\% (0,82-1)

Wiener-IICS: 0,89 IC95\% $(0,76-1)$

BiosChile-Wiener: 0,85 IC95\% $(0,6-1,1)$ 


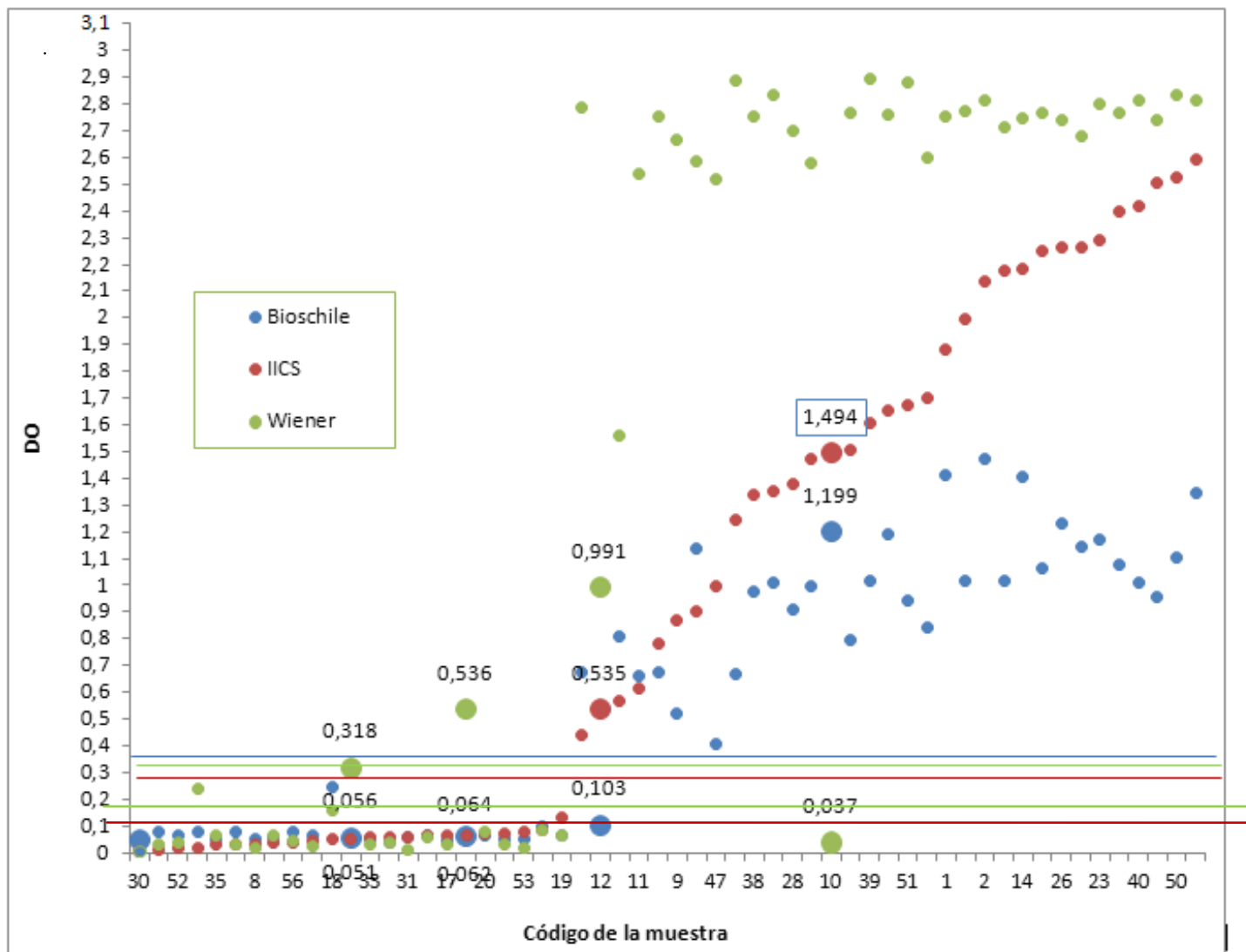

Figura 1. Distribución de la Desviación Óptica (DO) de las muestras por los tres kits utilizados en el estudio y sus respectivos puntos de corte (CO)

En cuanto a la evaluación externa de las 450 muestras analizadas, 372 fueron negativas y 78 positivas para la enfermedad de Chagas, obteniéndose una calificación " $A$ ", dentro del "Programa de Evaluación Externa de Desempeño en Serología" de la Fundação PróSangue/Hemocentro de São Paulo

En la evaluación interna, la media de los valores de la relación de positividad DO /CO del suero control interno fue de 4,5+/- 0,59 y el CV de 13\%. En la figura 2 se observa la distribución de los valores de los promedio por año.

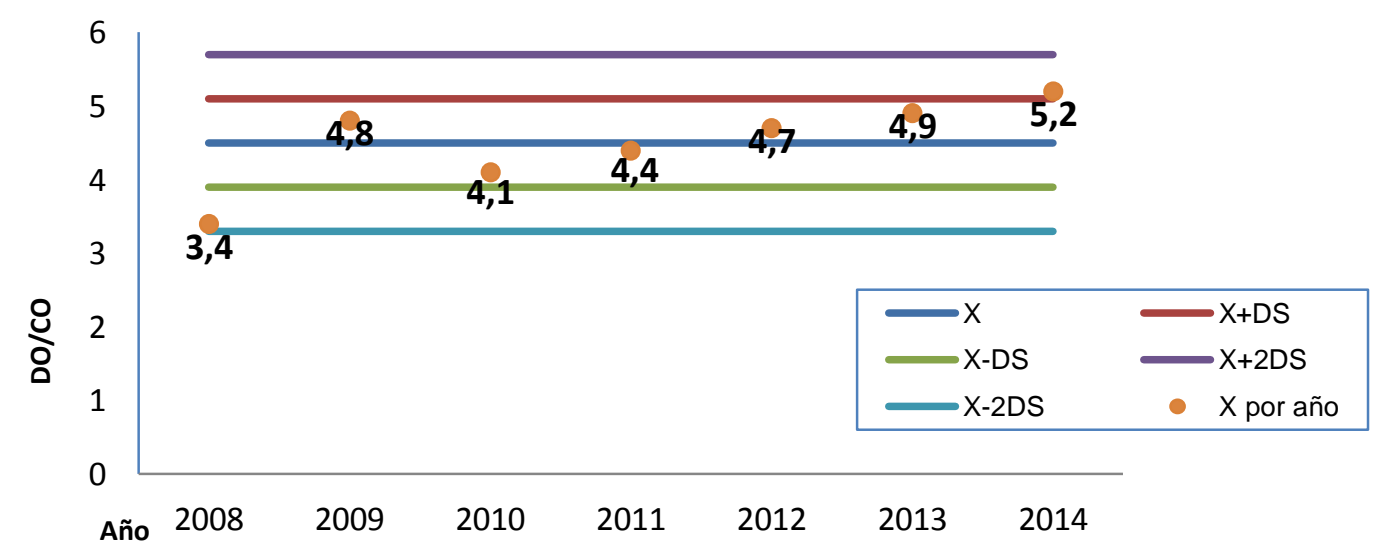

Figura 2. Promedio (X) de los valores del índice DO/CO y desvío estándar (DS) de los valores del control interno por año.

\section{DISCUSIÓN}

En este estudio se comparó la capacidad de detección de anticuerpos anti T. cruzi del Kit de Chagas test IICS V.1, con los kits comerciales Chagastest ELISA (Wiener) y con el 
BiosChile Test ELISA para Chagas III y hemos encontrado excelente concordancia con estos kits, que son utilizados en laboratorios públicos y privados de nuestro país por su alta sensibilidad y especificidad mayor al $99 \%$ (24). Los discordantes obtenidos en el estudio pudieran deberse a los distintos tipos de antígenos utilizados por los ELISAs, (25) lo cual se debería ser confirmado por otro método.

El método ideal es aquel que alcanza exactitud en los análisis y la mantiene a través del tiempo (26), los resultados obtenidos con el ELISA Chagas test en el "Programa de Evaluación Externa de Desempeño en Serología" de la Fundação Pró-Sangre/Hemocentro de São Paulo fueron óptimos, no presentando falsos positivos ni falsos negativos en más de 10 años de evaluación avalando como confiable para el diagnóstico. Los métodos basados en enzimoinmunoanálisis (ELISA) muestran un mejor desempeño que los métodos basados en técnicas de aglutinación o inmunofluorescencia dado que dependen de la destreza del operador y de la subjetividad en la lectura (16). Además ha demostrado tener una muy buena concordancia con los demás métodos utilizados en el programa de evaluación externa en forma indirecta ya que este programa evaluó las muestras enviadas por varios métodos de diferentes fundamentos tales como hemaglutinación indirecta (HAI) o la inmunofluorescencia indirecta (IFI) y otros ELISAs para el diagnóstico de Chagas.

Con respecto a la evaluación del suero control interno, en el kit de Chagas test IICS V.1 presenta buena precisión y exactitud los valores promedios obtenidos cada año fue de un CV del $13 \%$, muy por debajo del límite superior del $20 \%$ recomendado por el control de calidad en tamizajes de muestras de banco de sangre (27) indicando una buena precisión del kit.

La participación en programas de evaluación externa de calidad e implementación de un control de calidad interno y buenas practicas del laboratorio ha permitido alcanzar un nivel optimo en el desempeño del Kit de Chagas test IICS V.1.

La implementación de un kit de diagnóstico en el laboratorio de análisis clínico requiere la evaluación previa del mismo, a fin de establecer su desempeño y, por ende su aptitud para ser utilizado de manera segura y confiable $(28,29)$.El Chagas test IICS V.1 ha demostrado reunir los requisitos necesarios para ser empleado en el diagnóstico de la enfermedad, además de ser el único test de fabricación Nacional que cuenta el país para esta enfermedad lo cual facilita la adquisición continua del mismo para los laboratorios a nivel nacional y de aplicación sencilla para todos los niveles de complejidad laboratorial tanto en el interior como en la capital del país, sin embargo se ha visto la necesidad de seguir implementando mejoras al kit para que este pueda ser también aplicados a sistemas automatizados.

\section{REFERENCIAS BIBLIOGRÁFICAS}

1. Silveira AC. Lineamientos $y$ recomendaciones técnicas y de política pública para el abordaje de la enfermedad de Chagas. Programa regional para el control de la enfermedad de Chagas en América latina. Pág. 7

2. Dubner S, Schapachnik E, Riera AR, Valero E. Chagas disease: state-of-theart of diagnosis and management. Cardiol J. $2008 ; 15(6): 493-504$.

3. OPS. Enfermedad de Chagas. Guía para vigilancia, prevención, control y manejo clínico de la enfermedad de Chagas aguda transmitida por alimentos. Río de Janeiro: PANAFTOSA-VP/OPAS/OMS, 2009.

4. Rojas de Arias, A. Manejo de las vías alternativas de transmisión. La enfermedad de Chagas a la puerta de los 100 años del conocimiento de una endemia americana ancestral. OPS/CD/426-06. Buenos Aires, Fundación Mundo Sano; Julio 2007: 179 - 86.

5. OPS. Conceptualización de la vigilancia epidemiológica. Taller del Cono Sur sobre
Enfermedad de Chagas.Buenos Aires, Argentina, Setiembre 2003 - Montevideo 2004. OPS/DPC/CD/290/04.

6. Schumis GA. La tripanosomiasis americana como problema de salud pública. En: La enfermedad de Chagas y el sistema nervioso. Washington, D.C.: Organización Panamericana de la Salud (Publicación científica núm.547), 1994:331.

7. OPS. Estándares de Trabajo para Bancos de Sangre. Tercera Edición. Washington, DC: OPS; 2012.

8. ReglamentoTécnico Mercosur de Medicina Transfusional. Decreto No 385/000 MERCOSUR.PROMULGACION: 26 de diciembre de 2000. PUBLICACION: 9 de enero de 2001

9. WHO. (2002). Control of Chagas Disease [Documento en línea]. Disponible: http://whqlibdoc.who.int/trs/WHO_TRS_9 05.pdf

10. Behrend $M$, Beltrán $M$, Restrepo $M$, Kroeger A. Control de la enfermedad de chagas en bancos de sangre de 
Aria et al Desempeño del Kit ELISA Chagas IICS V.1para el diagnóstico de la enfermedad de Chagas Colombia. Biomédica. 2002; 22(1): 3945

11. Kaspar P, Velázquez G, Monzón MI, Meza $T$, Vera ME, Pozzoli L et al. Un Nuevo Kit para la determinación de anticuerpos anti-Tripanosoma Cruzi. En: Rosner JM, Kawabata M, editores. Enfermedad de Chagas en el Paraguay. Asunción: Memorias del IICS No 15. EFACIM-JICA; 1990.

12. Russomando G, Almirón M, Candía N, Franco L, Sánchez $\mathrm{z}$, Guillen I. Implementación y evaluación de un sistema localmente sustentable de diagnóstico prenatal que permite detectar casos de transmisión congénita de la enfermedad de Chagas en zonas endémicas del Paraguay. Revista da Sociedade Brasileira de Medicina Tropical. 2005 38:49-54

13. Russomando G. Transmisión congénita de la enfermedad de Chagas en Paraguay. Mem. Inst. Investig. Cienc Salud. 2009: 55-64.

14. Lorca M, Child R, Garcia A, Silva MG, Martínez LP, Jerez GM, Toledol, Mezzano DA. 1994. Evaluación de reactivos comerciales empleados en el diagnóstico de la enfermedad de Chagas en bancos de sangre de Chile. II Aplicación rutinaria. Rev.Med.Chile 122:925-31

15. Bellmunt-Montoya S. Validación de Pruebas Diagnósticas. Angiología 2007; 59 (6): 433-38.

16. Illa C, D'Agostino L, Willie JC, Mazziotta D. Evaluación externa de calidad para el diagnóstico serológico de la enfermedad de Chagas: 3 años de evolución. Acta bioquím. clín. latinoam. [revista en la Internet]. 2005 Sep [citado 2010 Mayo 08]; 39(3): 355-57. Disponible en: http://www.scielo.org.ar/scielo.php?scrip $\mathrm{t}=$ sci_arttext\&pid=S0325-

9572005000300011\&lng=es $>$.

17. Sáez-Alquézar A, Otani $M$, Sabino EC, Salles NA, Chamone DF. Programas de control externo de la calidad en serología, desarrollados en América Latina con el apoyo de la OPS entre 1997 y 2000. RevPanamSaludPublica. 2003;13(23):91-102.

18. Otani M, Vinelli E, Kirchhoff L, Pozo A, Sands A et al. WHO comparative evaluation of serologic assays for chagas desease. Articulo Original. Transfusion 2009.

19. Landis JR, Koch GG. The measurement of observer agreement for categorical data. Biometrics 1977 33: 159-74.

20. Tellez W. Peñaloza R. Aspectos metodológicos del control de calidad intralaboratorio en Química Sanguinea.WWW.ops,org.bo.

21. Sáez-Alquezar A, Albajar-Viñas $P$, Valpassos Guimarães A, Abol Corrêa J. Control de calidad en el tamizaje para enfermedades infecciosas en bancos de 